M. A. Smith, H. F. Henrichs, and J. Fabnegat, eds.

\title{
BCD Classification of Stars with the B[e] Phenomenon ${ }^{1}$
}

\section{Cidale}

Observatorio de La Plata, Paseo del Bosque $S / N, 1900$ La Plata, Argentina

\section{J. Zorec}

Institut d'Astrophysique de Paris, CNRS, $98^{\text {fbis }}$ bd. Arago, F-75014 Paris, France

\section{N. Morrell}

Observatorio de La Plata, Paseo del Bosque S/N, 1900 La Plata, Argentina

\begin{abstract}
Stars in different evolutionary stages and/or environments can show the $\mathrm{B}[\mathrm{e}]$ phenomenon. In order to establish possible relations between the stellar evolutionary stage and the presence of this phenomenon, the fundamental parameters of these stars need to be determined, as in most cases they are unknown. With this aim we undertook systematic low resolution spectroscopic observations of stars with the $\mathrm{B}[\mathrm{e}]$ phenomenon. Preliminary determinations of fundamental parameters based on the BCD (Barbier-Chalonge-Divan Paris spectrophotometric system) analysis are reported in this paper.
\end{abstract}

\section{Introduction}

Some B and late $\mathrm{O}$ type stars with hydrogen emission lines also show forbidden emission lines in the visual spectral range. In most cases, the presence of forbidden lines is correlated with a strong IR flux excess (Allen \& Swings 1976). Although they all have the generic name of "B[e] stars", a large variety of objects may in principle be comprised in this group. A quick look at the existing lists of known B[e] stars (Jaschek \& Egret 1982) reveals that the "B[e] phenomenon" concerns objects that may also show properties of "classic" Be stars (Jaschek et al. 1981), as well as those of supergiants, planetary nebulae, symbiotic and young stellar objects either in the early main sequence or in a pre-main sequence evolutionary stage. This may imply that the circumstellar envelopes giving rise to the line and continuum emissions were formed by different mechanisms and/or that the "B[e] phenomenon" concerns a different type of object. Qualitative information on the underlying stars showing the "B $[\mathrm{e}]$ phenomenon" may in part

\footnotetext{
${ }^{1}$ Data obtained in CASLEO operated under agreement between the CONICET and the Universities of La Plata, Córdoba and San Juan, Argentina
} 
be obtained from the analysis of their continuum energy distribution around the Balmer discontinuity. It is known that the Balmer discontinuity provides information on the effective temperature of the central star and its surface gravity. Thus, the main concern of this paper is the study of the Balmer discontinuity of stars showing the $\mathrm{B}[\mathrm{e}]$ phenomenon.

\section{Formal classification of stars with the $B[e]$ phenomenon}

In a recent paper (Lamers et al. 1998) it was proposed that it is better to refer to the " $\mathrm{B}[\mathrm{e}]$ phenomenon" rather than to use the name " $\mathrm{B}[\mathrm{e}]$ stars". In that paper, five classes of stars which show the $B[e]$ phenomenon were identified according to different physical characteristics of the central stars and the circumstellar matter:

1. $\mathrm{B}[\mathrm{e}]$ supergiants or "sgB[e]"

2. Pre-main sequence B[e]-type stars or "HAeB[e] stars"

3. Compact planetary nebulae B[e]-type stars or "cPNB $[\mathrm{e}]$ stars"

4. Symbiotic B[e]-type stars or "SymbioticB[e] stars"

5. Unclassified B[e]-type stars or "unclB[e] stars"

As a number of objects satisfy the criteria for more than one of the above classes, their evolutionary phases are unclear and the stars are considered "unclB[e] stars".

\section{The observations}

The first set of spectroscopic observations were obtained in October 1997 and August 1998 at the Complejo Astronómico El Leoncito (CASLEO), Argentina. We used the Boller \& Chivens Cassegrain spectrograph with a 600 lines $\mathrm{mm}^{-1}$ grating and a $512 \times 512 \mathrm{CCD}$ detector attached to the $2.15 \mathrm{~m}$ telescope. The observed spectral range is $\lambda \lambda 3500-4600 \AA$ where the 2 pixel resolution represents $4.53 \AA$. Reductions were performed by the IRAF software package (Version 2.10.2). All extracted spectra were wavelength and flux calibrated, and were also corrected for atmospheric extinction. The task "splot" was used to obtain $\log F_{\lambda}$ vs. $\lambda$ and $\log F_{\lambda} / b_{\lambda}$ vs. $1 / \lambda$ plots $\left(b_{\lambda}\right.$ is the black-body function for a given temperature normalized to its peak intensity).

We have observed stars with the $\mathrm{B}[\mathrm{e}]$ phenomenon in almost all classes discussed in Sect. 2. However, the results presented here refer only to some of the observed stars, including several of the HAeBe type. The set of stars displayed in this contribution aims at presenting a testing step of a systematic classification program of this type of objects, which is currently performed in the BCD system.

\section{The $\mathrm{BCD}$ system}

The BCD (Barbier-Chalonge-Divan) spectrophotometric classification system was developed at the Institut d'Astrophysique de Paris by Barbier \& Chalonge (1941) and Chalonge \& Divan (1954). To perform stellar classification in the BCD system, low resolution spectra are suitable at least in the $\lambda \lambda 3500-4600$ 

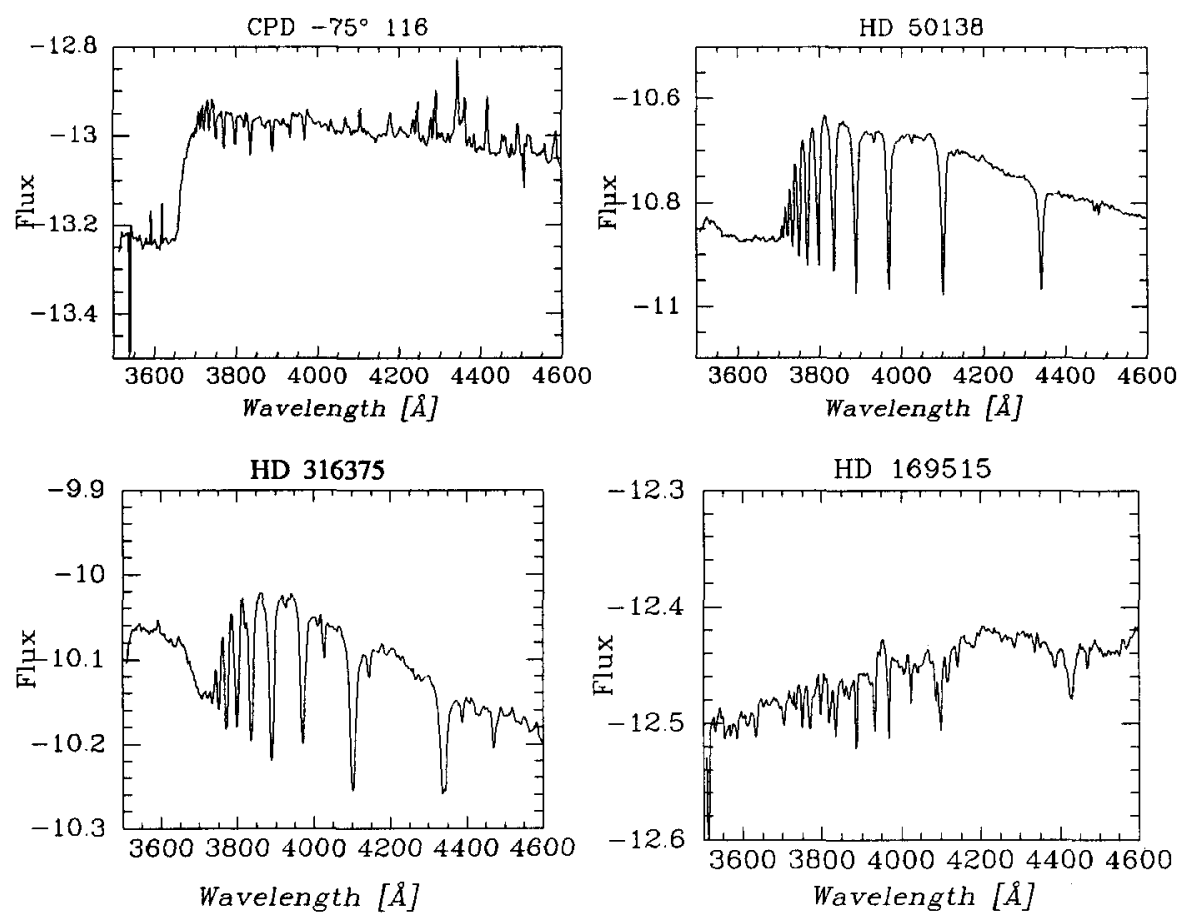

Figure 1. Low resolution spectra near the Balmer discontinuity of four stars with different formal "B[e] classification": CPD $-75^{\circ} 116$ (sgB[e]), HD 50138 (unclB[e]), HD 316375 (HAeB[e]) and HD 169515 (symB $[\mathrm{e}])$.

wavelength interval, so as to have a clear display of the Balmer discontinuity. In the original form of the BCD system, spectra were obtained in the $\lambda \lambda 3200-6500 \AA$ interval. This classification system is thus suitable for stars hotter than spectral type F9 and cooler than about 04 . Two parameters are derived: $D$ in dex, the Balmer jump measured at $\lambda 3700 \AA$ which is obtained by extrapolating the Paschen continuum energy distribution in a $\log F_{\lambda} / F_{\lambda}^{*}$ vs. $1 / \lambda$ display, where $F_{\lambda}^{*}$ is a comparison flux distribution; $\lambda_{1}$, given in units of the difference $\lambda-3700 \AA . D$ is a strong indicator of the photospheric effective temperature and $\lambda_{1}$ of the luminosity class, so that we can easily derive the photospheric $\log g$ parameter. Stars with a circumstellar envelope, where the density is lower than photospheric density, show two Balmer discontinuities. As the second component (due to the circumstellar matter) is either in emission or in absorption and it is strongly shifted to the blue from the main or the stellar Balmer discontinuity, both components are clearly distinguished. The system is then suited also for spectral classification of abnormal stars, because the photospheric BCD parameters are not affected either by interstellar absorption or by 
circumstellar absorption/emission. On the other hand, as the $\left(\lambda_{1}, D\right)$ parameters are obtained from the continuum energy distribution they refer, on average, to deeper photospheric layers than any classification system based on spectral line measurements.

\section{Results}

The BCD classification for a small set of stars is given in Table 1. The effective temperature $T_{\text {eff }}$ and $\log g$ corresponding to the measured $\left(\lambda_{1}, D\right)$ are also given, as they would correspond to the photosphere of a normal OB star. They were derived using Divan \& Zorec's (1982) calibration of the $\left(\lambda_{1}, D\right)$ parameters in $T_{\text {eff }}$ and $\log g$.

The $T_{\text {eff }}$ obtained from the BCD calibrations are slightly lower than those obtained by Zickgraf et al. (1996). The values of $\lambda_{1}$ for these stars clearly indicate that they are supergiants of class Ia if the BCD classification is translated into the MK system. Our results for HD 45677 and HD 50138 are in good agreement with previous determinations for the same stars obtained from the original BCD system (Zorec et al. 1998, Lamers et al. 1998).

Table 1. Program stars classified in the BCD system

\begin{tabular}{|c|c|c|c|c|c|c|}
\hline Star & Ident. & $\begin{array}{l}\lambda_{1}-3700 \\
A A\end{array}$ & $\begin{array}{l}D \\
\text { dex }\end{array}$ & $\begin{array}{c}T_{\text {eff }} \\
10^{4} \mathrm{~K} \\
\end{array}$ & $\log g$ & Class \\
\hline CPD $-75^{\circ} 116$ & $\mathrm{~S} 65=\mathrm{R} 50$ & $11.1 \pm 4.7$ & $0.068 \pm 0.004$ & 1.28 & - & $\operatorname{sgB}[e]$ \\
\hline HDE 269217 & MWC 106 & $10.5 \pm$ & $0.082=$ & 1.2 & - & sgB \\
\hline $\mathrm{BD}-10^{\circ} 1351$ & AS 116 & $67.6 \pm 3.7$ & $0.181 \pm 0.012$ & 1.99 & 4.3 & HAeBe \\
\hline HD 45677 & MWC 142 & $54.8 \pm 1.6$ & $0.144 \pm 0.004$ & 2.19 & 3.9 & unclB $[\mathrm{e}]$ \\
\hline HD 50138 & MWC 158 & 41.7 & $0.295 \pm 0.004$ & 1.27 & 3. & unclB $[\mathbf{e}]$ \\
\hline HD 316375 & AS 246 & 63.0 & 0.164 & 2.10 & 4.2 & HAeBe \\
\hline HD 163296 & MWC 275 & $73.4 \pm 2.2$ & $0.434 \pm 0.006$ & 0.82 & 4. & HAAB \\
\hline HD 169515 & MWC 295 & $>100$ & $0.062 \pm 0.035$ & 3.20 & - & $\operatorname{symB}[e]$ \\
\hline BD $-11^{\circ} 4747$ & AS 321 & $66.8 \pm 4.2$ & $0.375 \pm 0.012$ & 1.15 & 4.2 & unclB $[\mathrm{e}]$ \\
\hline HD 190073 & MWC 325 & $50.4 \pm 0.3$ & $0.433 \pm 0.002$ & 1.11 & 3.7 & $\mathrm{HAeBe}$ \\
\hline
\end{tabular}

\section{References}

Allen, D.A., Swings, J.P. 1976, A\&A 47, 293

Barbier, D., Chalonge, D. 1941, Ann. d'Astrophys. 4, 30

Chalonge, \& Divan, 1952, Ann. d'Astrophys. 15, 201

Divan, L., Zorec, J. 1982, ESA-SP 177

Jaschek, M., Egret, D. 1982, CDS-SP No 4, Observatoire de Strasbourg

Jaschek, M., Slettebak, A., Jaschek, C. 1981, Be Stars Newsletter No 4, p.9

Lamers, H.J.G.L.M., Zickgraf, F.J., de Winter, D., Houziaux, L., Zorec, J. 1998, A\&A 340, 117

Zickgraf, F.J., Humphreys, R.M., Lamers, H.J.G.L.M. et al. 1996, A\&A 315, 510

Zorec, J., Moujtahid, A., Ballereau, D., Chauville, J. 1998, In: B[e] stars, (eds) A.M. Hubert \& C. Jaschek, Kluwer Ac. Pub. p. 55 